\title{
Symmetrization of the Non-rigid Registration Problem Using Inversion-Invariant Energies: Application to Multiple Sclerosis
}

\author{
Pascal Cachier and David Rey \\ INRIA Sophia - Epidaure Project \\ 2004 Route des Lucioles BP 93 \\ 06902 Sophia Antipolis Cedex, France \\ \{Pascal.Cachier, David.Rey\}@sophia.inria.fr
}

\begin{abstract}
Without any prior knowledge, the non-rigid registration of two images is a symmetric problem, i.e. we expect to find inverse results if we exchange these images. This symmetry is nonetheless broken in most of intensity-based algorithms. In this paper, we explain the reasons why most non-rigid registration algorithms are asymmetric. We show that the asymmetry of quadratic regularization energies causes an oversmoothing of expending regions relatively to shrinking regions, hampering in particular registration-based detection of evolving processes. We therefore propose to use an inversion-invariant energy to symmetrize the registration problem. To minimize this energy, two methods are used, depending on whether we compute the inverse transformation or not. Finally, we illustrate the interest of the theory using both synthetic and real data, in particular to improve the detection and segmentation of evolving lesions in MR images of patients suffering from multiple sclerosis.
\end{abstract}

\section{Introduction}

Non-rigid registration of two images $I$ and $J$ consists in finding a transformation between the two sets of points of both images. If we make the assumption that every point of an image has an homologous point in the other, and that the topology has been conserved, the result of the algorithm should be a smooth homeomorphic transformation $T(I, J)$, going from $I$ to $J$.

Among non-rigid registration algorithms, intensity-based algorithms are driven by a similarity energy $E_{\text {sim }}$ (e.g. the sum of square differences (SSD)) that attracts both images toward each other using their intensity values [1]. At the same time, the transformation $T(I, J)$ is kept smooth either by constraining $T(I, J)$ to belong to some space $\mathcal{T}$ of regular transformations (e.g. splines with few control points [2]), or by choosing a regularization energy $E_{\text {reg }}$ (e.g. the linear elastic energy), or both [3]. This energy $E_{r e g}$ can be minimized with $E_{\text {sim }}$ in a weighted sum $E_{\text {sim }}+\lambda E_{\text {reg }}[4,5]$, or minimized alternatively with $E_{\text {sim }}[6]$. See [7] for a review of registration techniques. 


\subsection{Asymmetry and Related Problems}

Without any other prior knowledge, the registration problem is symmetric and we expect to obtain the inverse transformation if we exchange $I$ and $J: T(J, I)=$ $[T(I, J)]^{-1}$. However, this symmetry is usually broken in practice, giving birth to the terminology "reference" or "template" image and "study" or "floating" image (here $T(I, J)$ goes from the reference image $I$ to the study image $J$ ). Most, if not all, of the energies used in medical image non-rigid registration leads to asymmetrical registration. This includes the usual similarity energies and quadratic regularization energies such as linear elastic or thin plate energies. Even the general, non-linear elasticity is conceptually asymmetric since one of the images is supposed to be in a no-stress state and not the other; see [8] for an example showing the importance of this consideration.

The first obvious problem with asymmetry is that we have to choose arbitrarily a reference image, and thus the results of measures relying on this registration depend on how the reference image is chosen.

However, there can be a more subtle problem than the previous one. Indeed, using standard similarity measures and quadratic regularization energies will lead to systematically biased solutions: they will smooth the transformation on expanding regions more than on shrinking regions. Both $T(I, J)$ and $T(J, I)$ will have more difficulty to describe a local growth than a local shrink.

In order to understand this, let us present a 1D example where we smooth a function $f$ using a convolution with a kernel of some finite extension $\sigma$ : we use values of $f$ in an interval of length $\sigma$ to smooth $f$. As shown on fig. 1 , the same smoothing is approximately obtained on $f^{-1}$ if it is convolved with a kernel of size $\sigma f^{\prime}$. Thus, the transformation around an expanding region $\left(f^{\prime} i 1\right)$ would be less smoothed if the images were exchanged and the inverse transformation smoothed with the same kernel of size $\sigma$.

This explains why the detection

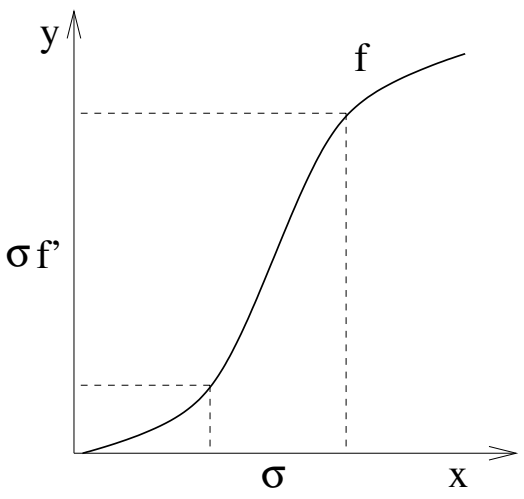

Fig. 1. Smoothing $f$ by a kernel of size $\sigma$ is roughly the same as smoothing $f^{-1}$ by a kernel of size $\sigma f^{\prime}$

of evolving brain lesions using non-rigid registration has appeared easier on shrinking lesions than on expanding lesions in our previous work [9]. We thus detected expanding and shrinking regions by working on shrinking regions of both $T(I, J)$ and $T(J, I)$.

\subsection{Towards Symmetry of Registration}

We have found that the asymmetry of intensity-based non-rigid registration is due to one or several of the following reasons: 
1. Order non-preservation of the energies: An energy $E(I, J, T)$ being used for registration, we may have $E\left(I, J, T_{1}\right)<E\left(I, J, T_{2}\right)$ but not $E\left(J, I, T_{1}^{-1}\right)<$ $E\left(J, I, T_{2}^{-1}\right)$ for two estimates $T_{1}$ and $T_{2}$ of the transformation.

2. Non-stable transformation space: The space of allowed transformation $\mathcal{T}$ may not be a group and thus not stable by inversion, i.e. we may have $T \in \mathcal{T}$ but not $T^{-1} \in \mathcal{T}$.

3. Local Minima: The optimization algorithm may get stuck in different local minima of the energy when the images are exchanged.

A previous work on the symmetrization of the registration has been done by Christensen [5]. In his work, two asymmetric registrations are made simultaneously, but are constrained to be close to the inverse of each other. This constraint is efficient at smoothing the registration. However, the algorithm is only asymptotically symmetric when the weight of this constraint tends towards infinity. Also, it relies on the computation of an inverse transformation whose existence is not ensured by linear elasticity. This work is nice but we felt that the symmetrization could be handled theoretically in a more efficient way.

In this paper, we propose to tackle the first point of the previous list and to ensure that the minima of the energy are invariant when exchanging the images, by forcing the similarity energy $E_{\text {sim }}$ and the smoothness energy $E_{\text {reg }}$ to be symmetric, i.e. invariant if we exchange $I$ and $J$ and invert the transformation: $E_{\text {sim }}(I, J, T)=E_{\text {sim }}\left(J, I, T^{-1}\right)$ and $E_{\text {reg }}(T)=E_{r e g}\left(T^{-1}\right)$. This is close to a very recent work by Trouv and Younes [10] where the authors consider distances of two parametric closed curves verifying some properties, including this kind of symmetry.

In section 2 we present a way to construct symmetric energies. In section 3 we present two optimization procedures, depending on whether we compute the inverse transformation or not. We illustrate the theory with a synthetic example in section 4 , and in section 5 we show the interest of this method to detect and segment evolving lesions in multiple sclerosis.

\section{Symmetric Energies}

\subsection{Symmetrization of Regularization Energies}

Let $\mathbf{x}$ be a point of $I$, and let $d^{n} T(\mathbf{x})$ be the $n$-th differential tensor of $T$ at $\mathbf{x}$. Let $E_{\text {reg }}(T)$ be a regularization energy that can be written as an integral of some function $e_{2}$ of the differentials of $T$ :

$$
E_{r e g}(T)=\int e_{2}\left(d T(\mathbf{x}), d^{2} T(\mathbf{x}), \ldots\right) d \mathbf{x}
$$

$E_{r e g}$ is symmetric (or inversion invariant) if $E_{r e g}(T)=E_{r e g}\left(T^{-1}\right)$. If we change variables in $E_{\text {reg }}\left(T^{-1}\right)$

$$
\begin{aligned}
E_{r e g}\left(T^{-1}\right) & =\int e_{2}\left(d\left(T^{-1}\right)(\mathbf{y}), d^{2}\left(T^{-1}\right)(\mathbf{y}), \ldots\right) d \mathbf{y} \\
& =\int e_{2}\left(d\left(T^{-1}\right) \circ T(\mathbf{x}), d^{2}\left(T^{-1}\right) \circ T(\mathbf{x}), \ldots\right)|d T(\mathbf{x})| d \mathbf{x}
\end{aligned}
$$


it appears that a sufficient condition for $E_{\text {reg }}$ to be symmetric is that

$$
e_{2}\left(d T(\mathbf{x}), d^{2} T(\mathbf{x}), \ldots\right)=e_{2}\left(d\left(T^{-1}\right) \circ T(\mathbf{x}), d^{2}\left(T^{-1}\right) \circ T(\mathbf{x}), \ldots\right)|d T(\mathbf{x})|
$$

where $|d T(\mathbf{x})|$ is the Jacobian of $T$ at point $\mathbf{x}$.

There are many such symmetric energies. Among them, we are more particularly interested in energies that are somehow linked to the asymmetric energies we are used to. There are several possibilities to symmetrize an asymmetric energy. Among them, the most natural is perhaps to take the mean of $e_{2}\left(d T, d^{2} T, \ldots\right)$ and $|d T| e_{2}\left(d\left(T^{-1}\right) \circ T, d^{2}\left(T^{-1}\right) \circ T, \ldots\right)$, i.e. the symmetrization $E_{\text {reg }}^{*}$ of a regularization energy $E_{\text {reg }}$ can be defined by

$$
E_{\text {reg }}^{*}(T)=\frac{1}{2}\left(E_{\text {reg }}(T)+E_{\text {reg }}\left(T^{-1}\right)\right)
$$

The trick is that $e_{2}\left(d\left(T^{-1}\right) \circ T, d^{2}\left(T^{-1}\right) \circ T, \ldots\right)$ can be calculate (more or less easily) from the derivatives of $T$. Thus, $E_{\text {reg }}^{*}(T)$ is indeed of the form (1) and can be computed without inverting $T$. For example, let us consider the following quadratic energy in 2D: $E_{r e g}(T)=\int_{\mathbf{R}^{2}}\|d T\|^{2}=\int_{\mathbf{R}^{2}}\left\|\partial_{x} T\right\|^{2}+\left\|\partial_{y} T\right\|^{2}$. Since $d\left(T^{-1}\right) \circ T=(d T)^{-1}$, we find that the symmetrization of this energy using eq. (2) is:

$$
E_{\text {reg }}^{*}(T)=\frac{1}{2} \int\left(1+\frac{1}{|d T|}\right)\left(\left\|\partial_{x} T\right\|^{2}+\left\|\partial_{y} T\right\|^{2}\right)
$$

Note that the integrand tends to $+\infty$ when $d T$ tends to 0 : in practice, this will force the transformation to be one-to-one.

\subsection{Symmetrization of Similarity Energies}

Similarity measures can be symmetrized using the same technique. If $E_{\operatorname{sim}}(I, J, T)$ is a similarity energy, a symmetric similarity energy can be deduced by setting:

$$
E_{\text {sim }}^{*}(I, J, T)=\frac{1}{2}\left(E_{\text {sim }}(I, J, T)+E_{\text {sim }}\left(J, I, T^{-1}\right)\right)
$$

Here again, changing the variables inside $E_{\text {sim }}\left(J, I, T^{-1}\right)$ usually eliminates $T^{-1}$ from the formulas, and therefore the inversion of $T$ can be avoided. For example, if $E_{\text {sim }}(I, J, T)=\int(I-J \circ T)^{2}$ is the standard SSD criterion between two images, then its symmetrization using (4) is

$$
E_{\text {sim }}^{*}(I, J, T)=\frac{1}{2} \int(1+|d T|)(I-J \circ T)^{2}
$$




\section{Optimization of the Symmetric Energy}

\subsection{Finite Element Implementation without Inversion}

Our first implementation uses the fact that the symmetric energies can be computed and optimized without inverting the displacement field. To produce the results presented in the next section, we have minimized in $2 \mathrm{D}$ the symmetric energy (7) which is the symmetrization of (6):

$$
\begin{aligned}
E(I, J, T) & =\int(I-J \circ T)^{2}+\lambda \int\|d T\|^{2} \\
E^{*}(I, J, T) & =\frac{1}{2}\left[\int(1+|d T|)(I-J \circ T)^{2}+\lambda \int\left(1+\frac{1}{|d T|}\right)\|d T\|^{2}\right]
\end{aligned}
$$

In our experiences, the accuracy of the symmetry has been very sensitive to the discretization of the continuous optimization problem. We found out that results were significantly better using a finite element discretization of the energy rather than a finite difference scheme. A triangularization of the rectangular grid defined by the pixels is used. The images as well as the transformation are defined as piecewise linear functions on this triangularization. To minimize the energy, we use a simple gradient descent coupled with a multiresolution scheme.

We encountered two types of problems with the previous algorithm:

1. The energy is difficult to minimize, mainly because it includes the term $1 /|d T|$ that raises numerical problems, especially when the smoothness constraint is set low $(\lambda \ll 1)$.

2. The optimization by gradient descent is asymmetric (point 3 of the list given in introduction): for example, the derivative of the energy (7) with respect to $T$ uses the derivative of $J$ and not the derivative of $I$. This hampers a perfect symmetry of the result.

\subsection{Alternate Minimization with Inversion}

These last problems can be avoided if we compute the inverse transformation. Our second method uses a very simple yet efficient alternated-minimization method: instead of minimizing $E^{*}(I, J, T)=E(I, J, T)+E\left(J, I, T^{-1}\right)$, we minimize alternatively the asymmetric energies $E(I, J, T)$ and $E\left(J, I, T^{-1}\right)$ (given by eq. (6)) using respectively $I$ and $J$ as the reference image. This requires an inversion of the transformation every time we change of reference image.

For a better comparison with the previous algorithm in 2D we have used the same finite element discretization. However, it is not necessary, which is one advantage of this method: faster optimizations are possible with the same quality of symmetry.

The inverse transformation is computed by finding the zero of $f_{\mathbf{y}}(\mathbf{x})=\mathbf{y}+$ $T(\mathbf{x})$ using a Newton scheme. Interpolation of the transformation is necessary; we have used a bi- or trilinear interpolation. The inverse is found with an error usually less than 0.1 voxel. Note that we cannot have an arbitrarily small error 
because the inverse of a piecewise bi/trilinear transformation is not piecewise bi/trilinear.

We have to be careful with the computation of the inverse. Indeed, $E(I, J, T)$ does not guarantee that the transformations is invertible. Forcing the corrections brought at each iteration to be small, and/or smoothing them, often helps the transformation to be invertible at every iteration, especially during the first iterations at full resolution when only a small number of points are moving. Furthermore, imposing small corrections seems to give more symmetrical results.

\section{Results on a Synthetic Example}

In this synthetic example we have two $520 \times 280$ images containing two discs, one multiplying its radius by 2 and the other shrinking symmetrically (fig. 3). We have registered these images using the asymmetric energy (6) (Asym algorithm), and using the algorithms described in sec. 3.1 (Sym1) and sec. 3.2 (Sym2).

When we register these images using Asym, we are confronted to the problem mentioned in sec. 1.1 that shrinkage is more easily recovered than growth. We can analyse it using the absolute value of the logarithm of the Jacobian (AVLJ) of the transformation, which expresses the local increase or decrease of area and we used for the detection of brain evolution in [9]. If the growth and the shrinkage were recovered equally, we would find equal AVLJ inside both

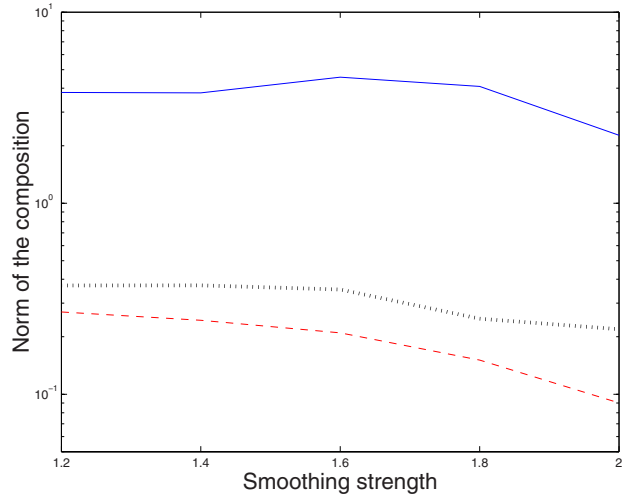

Fig. 2. Mean error in pixels of $T(I, J) \circ T(J, I)$ for Asym (plain), Sym1 (dots) and Sym2 (dashes). circles, and if they were furthermore exactly recovered, these numbers would be equal to $\log \left[\left(\pi(2 r)^{2}\right) /\left(\pi r^{2}\right)\right]=\log (4) \approx 1.39$. Instead, we find that both AVLJ are increasingly underestimated with the strength of the smoothing, which is expected, but also that the AVLJ of the expanding region is systematically underestimated relatively to the AVLJ of the shrinking region (fig. 3). This shortcoming is reduced with Sym1, and virtually eliminated with Sym2. We have also registered the images in both directions and computed $T(I, J) \circ T(J, I)$, which should be equal to identity in the case of a perfect symmetry. Mean errors of this composition relatively to the identity have been computed (fig. 2) and confirm the previous results.

We would like to emphasize that for the same amount of smoothing, Sym1 and Sym2 do not find a solution closer to the real transformation than the asymmetric algorithm for both circles: the small circle is registered better but the big 
circle is less shrinked. The distance to the real transformation is not uniformally decreased; errors are just shared more equitably.
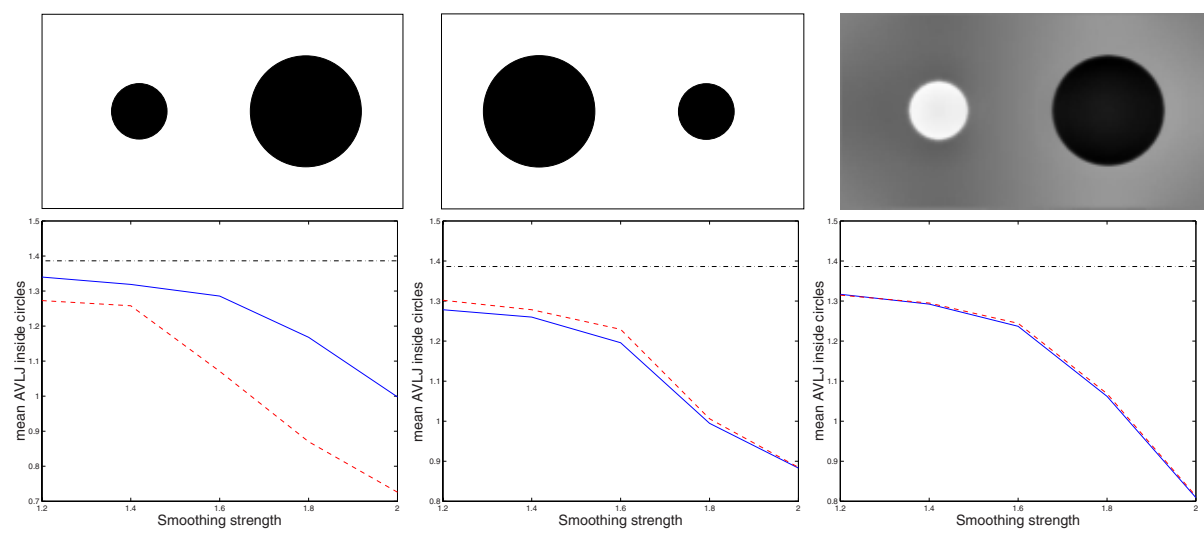

Fig. 3. Top: The two images to register (left and middle), and an example of a log-Jacobian image on the right (positive numbers are white, negative are black). Bottom: Graphs showing the mean absolute value of the log-Jacobian (AVLJ) inside the big circle (in plain) and inside the little circle (in dashed) relatively to the strength of the smoothing (x-axis), using respectively Asym (left), Sym1 (middle) and Sym2 (right). The real AVLJ is $\log (4) \approx 1.39$ (dashed-dotted line) for both circles.

\section{Application to Multiple Sclerosis}

In this section we would like to show that the method described in this paper can significantly improve results in the detection and segmentation of evolving lesions in multiple sclerosis [11, 9]. In a previous article [9] we explain that small expanding lesions can be difficult to detect with temporal analysis of apparent deformations: if there is an important expansion locally between images $I$ and $J$, we would need a one to many mapping due to limited resolution of the image. To avoid this, we considered only shrinking regions from $I$ to $J$, and then shrinking regions from $J$ to $I$. By thresholding shrinking areas we obtained the sought segmentations.

However it is possible to avoid the computation of 2 vector fields (direct and reverse) between images $I$ and $J$, by computing only one vector field thanks to a symmetrical approach. Here, we have used the alternated minimization method (sec. 3.2), without using a finite element scheme but the fast scheme of [6].

We have made some experiments on an evolving lesion extracted from two T2-weighted MRI of a patient with multiple sclerosis. Time between the two acquisitions is 8 weeks. The resolution of the images is really poor as we can see 
on the first two columns of figure 4 (voxel size is $0.9 \times 0.9 \times 5.5 \mathrm{~mm}^{3}$ ). We have computed four vector fields for our experiment: asymmetric and symmetric fields from $I$ to $J$, and asymmetric and symmetric fields from 2 to 1 . We compute the Jacobian on each field, and we manually search for the Jacobian isovalue (in red on fig. 4) that segments the lesion. As explained in [9], it does not exist any efficient automatic method to find the best threshold at the moment.

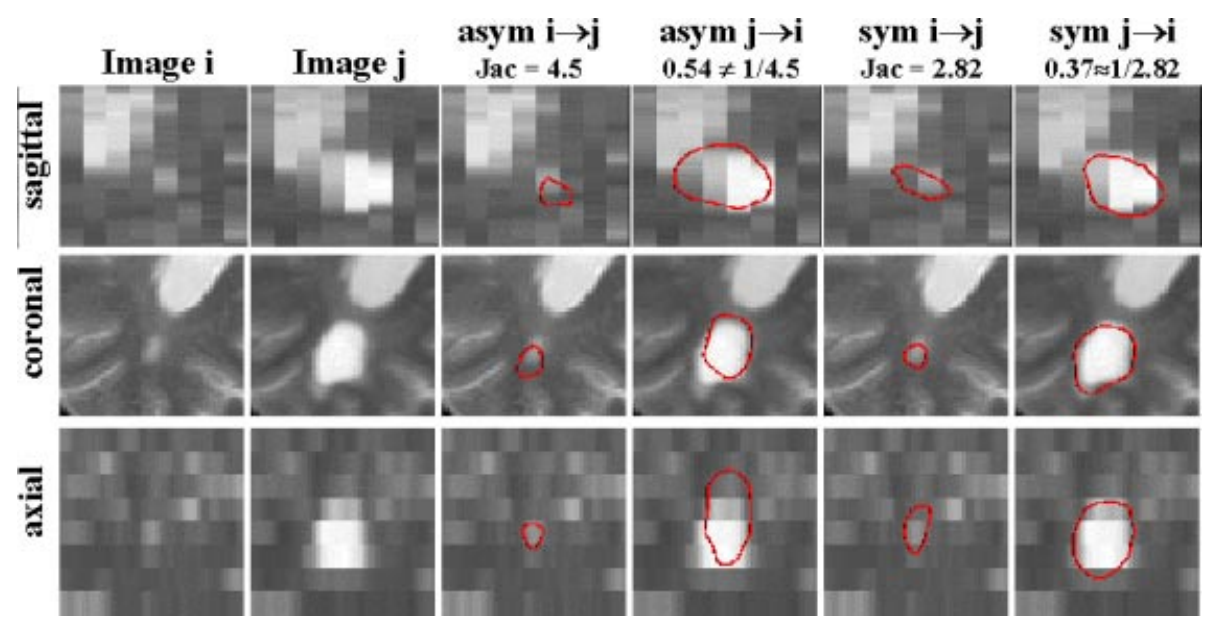

Fig. 4. Manual segmentations were done independently. Values are not inverse in the case of asymmetrical computation, and inverse in the case of symmetrical approach. Furthermore, the segmentation of evolving areas better corresponds to reality.

A symmetrical computation of the deformations significantly improves the detection and segmentation of evolving lesions. First it makes it possible to use the same value $v$ to threshold the Jacobian (Jac $>v>1$ for expansion, and Jac $<1 / v<1$ for shrinking). With an asymmetrical approach we had to compute the direct and reverse field and only search for shrinking areas. In figure 4 manual segmentations were done independently. Values are not inverse in the case of asymmetrical computation, and inverse in the case of symmetrical approach. Furthermore, the segmentation of evolving areas seems to better correspond to reality as we can see in figure 4 .

\section{Conclusion}

In this paper we have listed the reasons of the asymmetry of intensity-based registration algorithms. We have shown that asymmetry may hamper the equal retrieval of expanding and shrinking areas, perturbing registration-based detection of evolving processes. To reduce the asymmetry of registration, we have 
introduced inversion-invariant similarity and smoothness energies, and a way to symmetrize the widely used asymmetric energies.

We have worked on a particular asymmetric energy $E^{*}$ that is easily expressed as a function of the transform $T$. We have minimized $E^{*}$ with and without computation of the inverse transform. On a synthetic example, we have shown that the use of a symmetric energy was reducing considerably the shortcomings of asymmetric energies, and that the computation of the inverse transform, if it exists, is helpful. Finally, a symmetric approach improves segmentation results and simplify the methodology of registration-based detection of evolving lesions, especially in the case of small lesions and/or poor resolution.

\section{Acknowledgment}

This work was supported by the EC-funded BIOMORPH project 95-0845, a collaboration between the Universities of Kent and Oxford (UK), ETH Zürich (Switzerland), INRIA Sophia Antipolis (France) and KU Leven (Belgium) who provided us with multiple sclerosis images time series. Thanks to N. Ayache, X. Pennec and J. Stoeckel for proofreading and useful comments.

\section{References}

[1] A. Roche, G. Malandain, N. Ayache, and S. Prima. Towards a Better Comprehension of Similarity Measures Used in Medical Image Registration. In Proc. of MICCAI'99, pages 555 - 566, 1999.

[2] O. Musse, F. Heitz, and J.-P. Armspach. 3D Deformable Image Matching using Multiscale Minimization of Global Energy Functions. In Proc. of CVPR'99, 1999.

[3] D. Rueckert, C. Hayes, C. Studholme, P. Summers, M. Leach, and D. J. Hawkes. Non-Rigid Registration of Breast MR Images Using Mutual Information. In Proc. of MICCAI'98, pages 1144 - 1152, 1998.

[4] M. Ferrant, S. K. Warfield, C. R. G. Guttmann, R. V. Mulkern, F. A. Jolesz, and R. Kikinis. 3D Image Matching using a Finite Element Based Elastic Deformation Model. In Proc. of MICCAI'99, pages 202 - 209, 1999.

[5] G. E. Christensen. Consistent Linear-Elastic Transformations for Image Matching. In Proc. of IPMI'99, pages 224 - 237, 1999.

[6] X. Pennec, P. Cachier, and N. Ayache. Understanding the "Demons" Algorithm: 3D Non-Rigid Registration by Gradient Descent. In Proc. of MICCAI'99, pages $597-605,1999$. http://www.inria.fr/RRRT/RR-3706.html.

[7] J. B. A. Maintz and M. A. Viergever. A Survey of Medical Image Registration. Medical Image Analysis, 2(1):1-36, 1998.

[8] S. K. Kyriacou and C. Davatzikos. A Biomechanical Model of Soft Tissue Deformation, with Applications to Non-Rigid Registration of Brain Images with Tumor Pathology. In Proc. of MICCAI'98, pages 531 - 538, 1998.

[9] D. Rey, G. Subsol, H. Delingette, and N. Ayache. Automatic Detection and Segmentation of Evolving Processes in 3D Medical Images: Application to Multiple Sclerosis. In Proc. of IPMI'99, pages 154-167, 1999. http://www.inria.fr/RRRT/RR-3559.html. 
[10] A. Trouv and L. Younes. Diffeomorphicc Matching Problems in One Dimension: Designing and Minimizing Matching Functionals. In Proc. of ECCV'00 Part 1, pages 573-587, 2000.

[11] G. Gerig, D. Welti, C. Guttmann, A. Colchester, and G. Székely. Exploring the Discrimination Power of the Time Domain for Segmentation and Characterization of Lesions in Serial MR Data. In Proc. of MICCAI'98, pages 469-480, Boston, USA, 1998.

\section{A Appendix}

For a better comprehension, we develop the mathematics for obtaining the symmetric energies (3) and (5). If $T: \mathbf{R}^{2} \rightarrow \mathbf{R}^{2}$ is a diffeomorphic $2 \mathrm{D}$ transformation, we can compute its Jacobian matrix at a point $\mathbf{p}=(x, y)$ :

$$
d T(\mathbf{p})=\left(\frac{\partial T}{\partial x}(\mathbf{p}) \frac{\partial T}{\partial y}(\mathbf{p})\right)=\left(\begin{array}{c}
\frac{\partial T_{x}}{\partial x}(\mathbf{p}) \frac{\partial T_{x}}{\partial y}(\mathbf{p}) \\
\frac{\partial T_{y}}{\partial x}(\mathbf{p}) \frac{\partial T_{y}}{\partial y}(\mathbf{p})
\end{array}\right)
$$

Since $d\left(T^{-1}\right) \circ T=(d T)^{-1}$, we have

$$
d\left(T^{-1}\right)(\mathbf{q})=\frac{1}{|d T(\mathbf{p})|}\left(\begin{array}{cc}
\frac{\partial T_{y}}{\partial y}(\mathbf{p}) & -\frac{\partial T_{y}}{\partial x}(\mathbf{p}) \\
-\frac{\partial T_{x}}{\partial y}(\mathbf{p}) & \frac{\partial T_{x}}{\partial x}(\mathbf{p})
\end{array}\right)
$$

with $\mathbf{q}=T(\mathbf{p})$. Now, if

$$
E_{r e g}(T)=\int_{\mathbf{R}^{2}}\left(\frac{\partial T_{x}}{\partial x}(\mathbf{p})\right)^{2}+\left(\frac{\partial T_{x}}{\partial y}(\mathbf{p})\right)^{2}+\left(\frac{\partial T_{y}}{\partial x}(\mathbf{p})\right)^{2}+\left(\frac{\partial T_{y}}{\partial y}(\mathbf{p})\right)^{2} d \mathbf{p}
$$

then, using (8),

$$
\begin{aligned}
E_{r e g}\left(T^{-1}\right) & =\int_{\mathbf{R}^{2}} \frac{1}{|d T(\mathbf{p})|^{2}}\left[\left(\frac{\partial T_{x}}{\partial x}(\mathbf{p})\right)^{2}+\left(\frac{\partial T_{x}}{\partial y}(\mathbf{p})\right)^{2}+\left(\frac{\partial T_{y}}{\partial x}(\mathbf{p})\right)^{2}+\left(\frac{\partial T_{y}}{\partial y}(\mathbf{p})\right)^{2}\right] d \mathbf{q} \\
& =\int_{\mathbf{R}^{2}} \frac{1}{|d T(\mathbf{p})|}\left[\left(\frac{\partial T_{x}}{\partial x}(\mathbf{p})\right)^{2}+\left(\frac{\partial T_{x}}{\partial y}(\mathbf{p})\right)^{2}+\left(\frac{\partial T_{y}}{\partial x}(\mathbf{p})\right)^{2}+\left(\frac{\partial T_{y}}{\partial y}(\mathbf{p})\right)^{2}\right] d \mathbf{p}
\end{aligned}
$$

Finally we sum this last equation to $E_{\text {reg }}(T)$ to find the symmetrization of $E_{\text {reg }}(T)$ (eq. (3)).

Similarly, if $E_{\text {sim }}(I, J, T)=\int_{\mathbf{R}^{2}}(I(\mathbf{p})-J \circ T(\mathbf{p}))^{2} d \mathbf{p}$,

$$
\begin{aligned}
& E_{\text {sim }}\left(J, I, T^{-1}\right)=\int_{\mathbf{R}^{2}}\left(J(\mathbf{q})-I \circ T^{-1}(\mathbf{q})\right)^{2} d \mathbf{q} \\
& \stackrel{\mathbf{q}=T(\mathbf{p})}{=} \int_{\mathbf{R}^{2}}(J \circ T(\mathbf{p})-I(\mathbf{p}))|d T(\mathbf{p})| d \mathbf{p}
\end{aligned}
$$

We find eq. (5) by summing this last equation to $E_{\text {sim }}(T)$. 